\title{
MODELING GAS-PHASE TRANSPORT IN POLYMER-ELECTROLYTE FUEL CELLS
}

\begin{abstract}
A. Z. Weber and J. Newman
Lawrence Berkeley National Laboratory, Berkeley, CA 94720, USA

In this transaction, the equations and methodology for modeling convection and ordinary, Knudsen, and pressure diffusion of gases in a fuel-cell gas-diffusion layer are described. Some results examining the magnitudes of the various terms are also made. This derivation results in a self-consistent description of the various transport mechanisms and is robust for numerical solutions, especially for conditions involving different flow regimes or where the regime is not known a priori.
\end{abstract}

\section{$\underline{\text { Introduction }}$}

In fuel cells there are many different length scales, which allow for various scale separations. One example is the fact that the through-plane direction is often only coupled to the along-the-channel (in-plane) direction through material balances in the gas channel. Another example is the use of an agglomerate or reaction pellet in the catalyst layer, where the surface concentration is used to link the agglomerate scale with the layer length scale. A more complex example is that concerning diffusion in the various porous layers of the fuel cell. The issue is that there is both ordinary or Stefan-Maxwell diffusion along with Knudsen diffusion. The former is due to interactions between gas species and the latter due to interactions with the porous matrix. These two types of diffusion cannot be coupled by boundary conditions due to the fact that they occur throughout the medium. By an order-of-magnitude assessment, Knudsen diffusion is expected to become important when pore sizes are below a micrometer. While most fuelcell materials have pore sizes larger than this, catalyst and microporous layers often do not. Furthermore, there are also wide pore-size distributions for fuel-cell materials that often span into the submicrometer range. Finally, if a medium is hydrophobic, then the addition of liquid water results in the large pores being filled first, and thus the gas must move through the smaller ones, making Knudsen diffusion important.

This paper discusses how to couple the diffusive processes using a rigorous and consistent methodology. The approach also allows for the inclusion of convection and pressure diffusion in a consistent manner. Previous literature treatments of the various diffusive fluxes and convection have some shortcomings. ${ }^{1}$ The approaches utilizing dilute-solution theory do not account for all of the gas species' interactions among each other. The often-used dusty-gas model ${ }^{2}$ is based on unnecessary assumptions, such as assuming that the total flux is a linear combination of the viscous or convective flux and a diffusive flux. Finally, there are also piecemeal approaches that require changing matrices for different flow regimes so that a singular matrix does not develop. ${ }^{3}$ The approach presented in this paper circumvents such problems. 


\section{Derivation of Equations}

The analysis is begun with an $N$-component gas phase that interacts with a solid phase; therefore, it is a two-phase system with $N+1$ species ( $N$ gas species and the solid species). For each species, including the solid, momentum and material balances are written, and for the system, a total energy balance is written. For simplicity, an isothermal system is assumed, which takes the place of the energy balance. In addition, interstitial concentrations for the $N$ gas species are used, and an equation of state (e.g., the ideal-gas law in this paper) determines the pressure from the total concentration of these species. Such a treatment allows for the material balance for the solid species to become one of just specifying the porosity.

For the $N$ gas species, the material balances are relatively straightforward:

$$
\frac{1}{R T} \frac{\partial \varepsilon p_{i}}{\partial t}=-\nabla \cdot \mathbf{N}_{i}+R_{i}
$$

where $R_{i}$ represents a reaction term, $R$ is the ideal-gas constant, $T$ is the absolute temperature, $\varepsilon$ is the porosity of the system, $\mathbf{N}_{i}$ is the superficial flux density of species $i$, and $p_{i}$ is the interstitial partial pressure of species $i$. The term on the left side of the equation is the accumulation term, which accounts for the change in the total amount of species $i$ within a differential control volume. The first term on the right side of the equation keeps track of the material that enters or leaves the control volume by mass transport including both diffusion and convection, and the second term represents the generation (or consumption) of the material.

The momentum balances require a little more attention. Due to the small relaxation time for diffusive processes, the inertial terms are neglected in the momentum equations. By making this approximation, the momentum equations can be written as a balance between driving forces and frictional interactions,

$$
\mathbf{d}_{i}=\sum_{j \neq i} K_{i j}\left(\mathbf{v}_{j}-\mathbf{v}_{i}\right)
$$

where $\mathbf{d}_{i}$ is the negative of the driving force per unit volume acting on species $i$ (defined such that the summation of the frictional forces is zero), $K_{i j}$ is the frictional interaction parameter between species $i$ and $j$, and $\mathbf{v}_{i}$ is the velocity of $i$ relative to some reference velocity. $K_{i i}$ is undefined, and by the Onsager reciprocal relations $K_{i j}=K_{j i}$.

The left side of Eq. (2) can be replaced using the driving-force expression derived by Hirschfelder et $a l^{4}$

$$
\mathbf{d}_{i}=c_{i}\left[\nabla \mu_{i}-\frac{M_{i}}{\rho} \nabla p-\mathbf{X}_{i}+\frac{M_{i}}{\rho} \sum_{j} \mathbf{X}_{j} c_{j}\right]
$$

where $M_{i}$ is the molecular weight of species $i, \rho$ is the density of the solution, and the $\mathbf{X}_{i}$ terms refer to body forces per mole acting on species $i$ (e.g., gravity). In this discussion, body forces are neglected. One can then further simplify Eq. (3) by substituting in for the chemical potential and assuming ideal-gas behavior, 


$$
\mathbf{d}_{i}=\frac{y_{i} p}{R T}\left[R T \nabla \ln a_{i}+\overline{V_{i}} \nabla p-\frac{M_{i}}{\rho} \nabla p\right]=p \nabla y_{i}+\frac{y_{i} p}{R T}\left(\bar{V}_{i}-\frac{M_{i}}{\rho}\right) \nabla p
$$

where $\bar{V}_{i}$ is the partial molar volume of species $i$.

The right side of Eq. (2) can be written out taking the velocity of the solid as the reference velocity (this then serves as the momentum equation of the solid species),

$$
\begin{aligned}
\sum_{j \neq i} K_{i j}\left(\mathbf{v}_{j}-\mathbf{v}_{i}\right) & =\sum_{\substack{j \neq i \\
j \neq s}} \frac{p y_{i} y_{j}}{\frac{\varepsilon}{\tau} D_{i j}}\left(\mathbf{v}_{j}-\mathbf{v}_{i}\right)+\frac{p y_{i}}{\frac{\varepsilon}{\tau} D_{K_{i}}}\left(0-\mathbf{v}_{i}\right) \\
& =\sum_{\substack{j \neq i \\
j \neq s}} \frac{R T}{\varepsilon} D_{i j}\left(y_{i} \mathbf{N}_{j}-y_{j} \mathbf{N}_{i}\right)-\frac{R T}{\frac{\varepsilon}{\tau} D_{K_{i}}} \mathbf{N}_{i}
\end{aligned}
$$

where the subscript $s$ denotes the solid species. In the above expression, the frictional coefficients are related to diffusion coefficients, or parameters that vary inversely with momentum, by introducing a molar concentration scale. The interactions between gas species are denoted using a Stefan-Maxwell binary interaction parameter, and those with the solid using a Knudsen diffusion coefficient. ${ }^{5}$ In the above expression, the tortuousity and porosity corrections to the diffusion coefficients to account for the porous matrix are explicitly shown.

Equating Eqs. (3) and (4) results in the governing momentum equation for the gas species

$$
\nabla y_{i}=-\frac{y_{i}}{R T}\left(\bar{V}_{i}-\frac{M_{i}}{\rho}\right) \nabla p+\sum_{\substack{j \neq i \\ j \neq s}} \frac{R T}{p_{\frac{\varepsilon}{\tau}} D_{i j}}\left(y_{i} \mathbf{N}_{j}-y_{j} \mathbf{N}_{i}\right)-\frac{R T}{p_{\frac{\varepsilon}{\tau}} D_{K_{i}}} \mathbf{N}_{i}
$$

On the right side, the first term represents pressure diffusion, the second is ordinary diffusion, and the third is Knudsen diffusion. There are $N-1$ independent equations of the form of Eq. (6). One of the equations is dependent on the others through the GibbsDuhem equation or the fact that the sum of the mole fractions equals one.

To account for the gas-phase pressure drop, a convection equation is required. For porous media, Darcy's law is typically used: ${ }^{6}$

$$
\mathbf{v}=\frac{\sum_{i \neq s} M_{i} \mathbf{N}_{i}}{\rho}=-\frac{k}{\mu} \nabla p
$$

where $\mathbf{v}$ is the superficial mass-average velocity, $\mu$ is the viscosity, and $k$ is the permeability coefficient or Darcy's constant.

Doing an accounting of variables and equations, we see that we have: $N-1$ momentum equations of the form of Eq. (6); Darcy's law, Eq. (7); the stationary velocity or momentum equation of the solid); the porosity or material balance of the solid; $N$ material balances for the gaseous species of the form of Eq. (1); the sum of the interstitial partial pressures equals 1; and the assumption that the temperature is constant. This yields $2 N+4$ equations. In terms of variables, there are $N+1$ fluxes and $N+1$ concentrations (including those for the solid species), temperature, and pressure, yielding 
$2 N+4$ variables. Thus, we have the necessary amount of equations, and the system is properly specified.

Before proceeding to discuss some simulation results, comment should be made concerning the necessary $2 N+1$ boundary conditions. The temperature and pressure in the gas channel serve as conditions for those variables. For the flux variables, one can use the fact that the membrane is essentially impermeable to gas as a zero-flux condition at the membrane boundary ( $N$ conditions). For the concentration or mole fractions, one can do a material balance in the gas channel to determine them. This results in $N-1$ conditions (accounting for the fact that the sum of the mole fractions is unity).

\section{$\underline{\text { Discussion }}$}

It is of interest to examine some limiting cases and order of magnitudes of the various contributions to gas movement. In terms of limiting conditions, if the pressure is constant, then the equations result in a purely diffusive description of transport. The second limiting case is where Knudsen diffusion is not dominant but there is still convective motion caused by a pressure gradient. In this situation, the above expressions still hold and do not become ill-defined. The final and third limiting case is where there is neither convection nor Knudsen diffusion. This results in the Stefan-Maxwell equations for multicomponent diffusion. Overall, using the above set of equations does not result in a singular matrix regardless of the flow regime; they always remain valid.

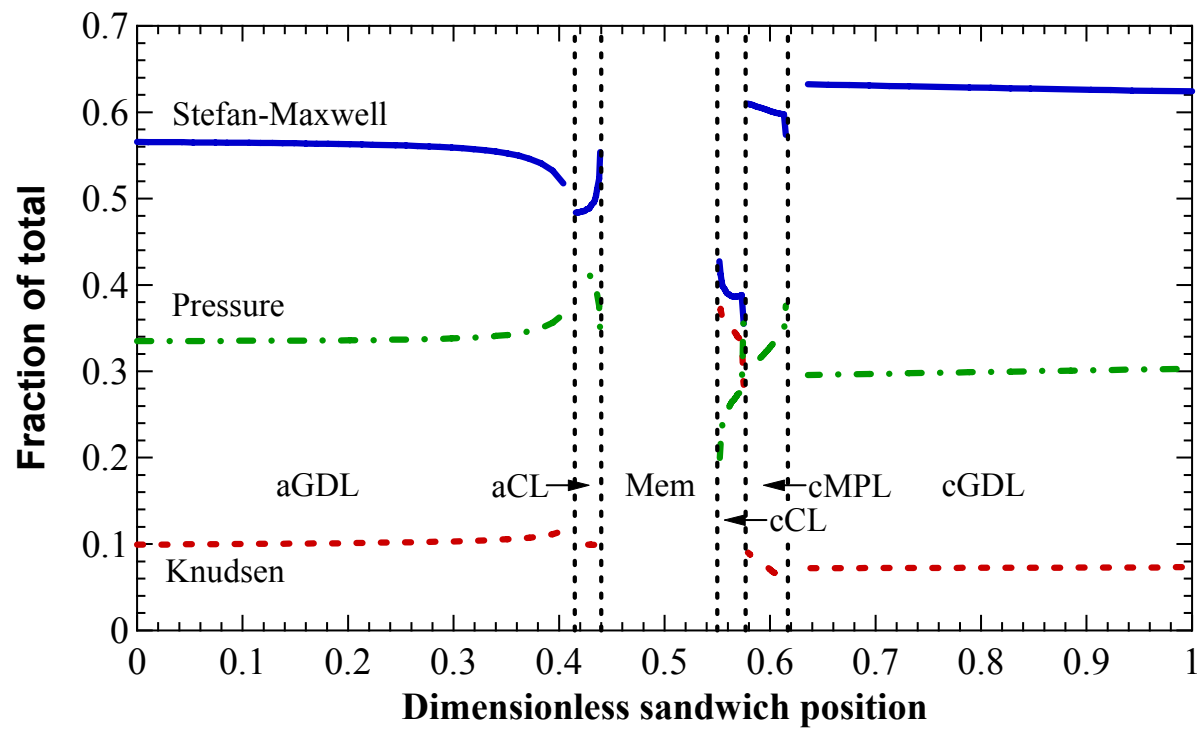

Figure 1. Fractional contributions to the hydrogen and oxygen mole fraction gradients on the anode and cathode side of the fuel cell, respectively. The three lines correspond to the three terms in Eq. (6). The simulation conditions are saturated feeds at $60^{\circ} \mathrm{C}$ and 1 bar and a current density of $0.9 \mathrm{~A} / \mathrm{cm}^{2}$.

To assess the relative importance of the various terms in Eq. (6) and pressure drop, simulations are run using our previously detailed 1-D fuel-cell-sandwich model. ${ }^{7-9}$ The modeling domain consists of symmetric anode and cathode gas-diffusion layers, membrane, cathode microporous layer, and anode and cathode catalyst layers. In the gas- 
diffusion layers, our two-phase-flow equations ${ }^{7}$ along with the above set for gas-phase transport are used. The use of two-phase flow alters the above equations in terms of the effective values of the diffusion coefficients (the porosity, tortuousity, and Knudsen diffusion coefficient can change), and the value of the gas-phase permeability.

The breakdown of the various contributions of the three terms on the right side of Eq. (6) are shown in Figure 1 for hydrogen (anode) and oxygen (cathode). The figure clearly demonstrates that all three of the diffusion types need to be accounted for. Knudsen diffusion is important mainly in the catalyst layers, as expected. However, pressure diffusion is shown to be important throughout the various porous media. This is surprising since pressure diffusion can normally be neglected in low-pressure systems. However, it is significant in the fuel cell due to the differences in molecular weight between water and hydrogen or oxygen, as well as the fact that the water partial pressure remains at its vapor pressure for these simulations.

Figure 1 demonstrates the importance of the various terms relative to each other, but one can still ask if they are important in terms of performance. For this analysis, polarization curves are generated as shown in Figure 2. While the base case utilized the full set of equations described above, the other curves subsequently remove different gasphase momentum mechanisms. The overall performance difference is only minor and is exhibited only in the mass-transfer regime of the polarization curve. Thus, while Figure 1 demonstrates that, when considered, the various contributions are each significant, Figure 2 demonstrates that they are not all important from an overall performance standpoint. Furthermore, although not shown here, the spread in the curves becomes less distinguishable as flooding becomes less important (i.e., with improved watermanagement schemes). In essence, these results demonstrate that the mole-fraction gradient is dependent on the overall system involving the various reactant fluxes and the saturated conditions, and not solely on the gas-phase momentum mechanism. However, it is more rigorous and advisable to use all of the terms in equations derived above.

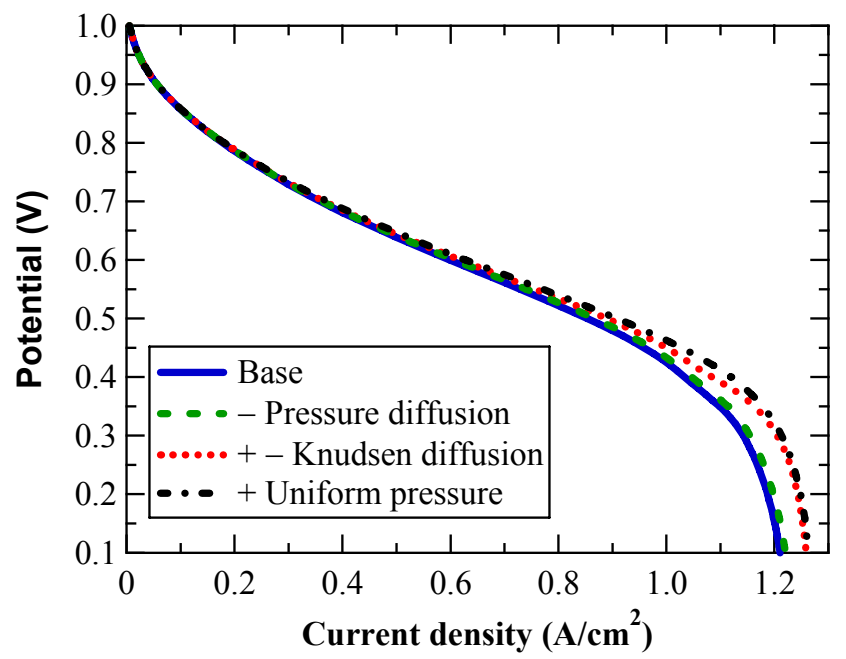

Figure 2. Polarization curves with gas-phase momentum contributions subsequently removed. The base case contains the set of equations above, the next removes pressure diffusion, then Knudsen diffusion is neglected, and finally a uniform gas pressure is assumed (i.e., infinite gasphase permeability). 


\section{$\underline{\text { Summary }}$}

A consistent set of equations has been developed that describes gas-phase transport in a fuel-cell porous medium. The phenomena involve convection, Knudsen diffusion, pressure diffusion, and ordinary or Stefan-Maxwell diffusion. The methodology used here is general and can be extended to systems that are nonisothermal, have body forces, or where the solid is reacting or moving. The derived set of equations results in a matrix of equations that remains well defined at all regimes of transport.

\section{Acknowledgement}

This work was supported by the Assistant Secretary for Energy Efficiency and Renewable Energy, Office of Hydrogen, Fuel Cell, and Infrastructure Technologies, of the U. S. Department of Energy under contract number DE-AC02-05CH11231.

\section{$\underline{\text { References }}$}

1. $\quad$ A. Z. Weber and J. Newman, Chem. Rev., 104, 4679 (2004).

2. E. A. Mason and A. P. Malinauskas, Gas Transport in Porous Media: The DustyGas Model, Elsevier, Amsterdam (1983).

3. A. A. Kulikovsky, J. Divisek, and A. A. Kornyshev, J. Electrochem. Soc., 146, 3981 (1999).

4. J. O. Hirschfelder, C. F. Curtiss, and R. B. Bird, Molecular Theory of Gases and Liquids, John Wiley \& Sons, Inc., New York (1954).

5. M. Knudsen, The Kinetic Theory of Gases, Methuen, London (1934).

6. F. A. L. Dullien, Porous Media: Fluid Transport and Pore Structure 2nd ed., Academic Press, Inc., New York (1992).

7. A. Z. Weber, R. M. Darling, and J. Newman, J. Electrochem. Soc., 151, A1715 (2004).

8. A. Z. Weber and J. Newman, J. Electrochem. Soc., 151, A311 (2004).

9. $\quad$ A. Z. Weber and J. Newman, J. Electrochem. Soc., 152, A677 (2005). 